\title{
Coulomb dissociation reactions on Mo isotopes for astrophysics applications*
}

\author{
O. Ershova ${ }^{\dagger 1,2}$, P. Adrich ${ }^{1}$, H. Alvarez-Pol ${ }^{3}$, F. Aksouh ${ }^{1}$, T. Aumann ${ }^{1}$, M. Babilon $^{4}$, \\ K.-H. Behr ${ }^{1}$, J. Benlliure ${ }^{3}$, T. Berg ${ }^{5}$, M. Böhmer ${ }^{6}$, K. Boretzky ${ }^{1}$, A. Brünle ${ }^{1}$, \\ R. Beyer ${ }^{11}$, E. Casarejos ${ }^{3}$, M. Chartier ${ }^{7}$, A. Chatillon ${ }^{1}$, D. Cortina-Gil ${ }^{3}$, U. Datta \\ Pramanik $^{8}$, L. Deveaux ${ }^{9}$, M. Elvers ${ }^{4,10}$, T. W. Elze ${ }^{2}$, H. Emling ${ }^{1}$, M. Erhard ${ }^{11}$, \\ B. Fernandez-Dominguez ${ }^{7}$, H. Geissel ${ }^{1}$, M. Gorska ${ }^{1}$, M. Heil ${ }^{1}$, M. Hellström ${ }^{1}$, \\ G. Ickert ${ }^{1}$, H. Johansson ${ }^{1,15}$, A. R. Junghans ${ }^{11}$, F. Käppeler ${ }^{18}$, O. Kiselev ${ }^{5}$, \\ A. Klimkiewicz ${ }^{1}$, J. V. Kratz ${ }^{5}$, R. Kulessa ${ }^{12}$, N. Kurz ${ }^{1}$, M. Labiche ${ }^{13}$, T. Le Bleis ${ }^{1,17}$, \\ R. Lemmon ${ }^{14}$, K. Lindenberg ${ }^{4}$, Y. Litvinov ${ }^{1}$, P. Maierbeck ${ }^{6}$, A. Movsesyann ${ }^{1,4}$, \\ S. Müller ${ }^{4}$, T. Nilsson ${ }^{15}$, C. Nociforo ${ }^{1}$, N. Paar ${ }^{16}$, R. Palit ${ }^{1}$, S. Paschalis ${ }^{7}$, R. Plag ${ }^{1,2}$, \\ W. Prokopowicz ${ }^{1}$, R. Reifarth ${ }^{1,2}$, D. Rossi ${ }^{5}$, L. Schnorrenberger ${ }^{4}$, H. Simon ${ }^{1}$, \\ K. Sümmerer ${ }^{1}$, G. Surowka ${ }^{12}$, D. Vretenar ${ }^{16}$, A. Wagner $^{11}$, S. Walter ${ }^{18}$, W. Walus ${ }^{12}$, \\ H. Weick ${ }^{1}$, N. Winckler ${ }^{18}$, M. Winkler ${ }^{1}$, A. Zilges ${ }^{4,10}$ \\ E-mail: o.ershova@gsi.de \\ ${ }^{1}$ GSI, Darmstadt, Germany; ${ }^{2}$ Univ. of Frankfurt, Germany; ${ }^{3}$ Univ. Santiago de Compostela, \\ Spain; ${ }^{4}$ TU Darmstadt, Germany; ${ }^{5}$ Univ. Mainz, Germany; ${ }^{6}$ TU München, Germany; ${ }^{7}$ Univ. \\ Liverpool, UK; ${ }^{8}$ SINP Kolkata, India; ${ }^{9}$ Univ. Orsay, France; ${ }^{10}$ Univ. zu Köln, Germany; ${ }^{11}$ FZ \\ Dresden-Rossendorf, Germany; ${ }^{12}$ Univ. Krakow, Poland; ${ }^{13}$ Univ. Paisley, UK; ${ }^{14}$ CCLRC \\ Daresbury Laboratory, UK, ${ }^{15}$ Chalmers University of Technology, Sweden; ${ }^{16}$ Univ. Zagreb, \\ Croatia; ${ }^{17}$ Univ. Strasbourg, France; ${ }^{18}$ FZ Karlsruhe, Germany.
}

\begin{abstract}
Coulomb excitation cross sections on the stable ${ }^{92,94,100} \mathrm{Mo}$ and unstable ${ }^{93} \mathrm{Mo}$ isotopes were measured at the SIS/FRS/LAND facility at GSI, Darmstadt. Experimental data on these isotopes are important to explain the problem of the underproduction of ${ }^{92} \mathrm{Mo}$ and ${ }^{96} \mathrm{Ru}$ in all existing models of p-process nucleosynthesis. Preliminary integral cross sections for ${ }^{100} \mathrm{Mo}(\gamma, \mathrm{n}){ }^{99} \mathrm{Mo},{ }^{100} \mathrm{Mo}(\gamma, 2 \mathrm{n}){ }^{98} \mathrm{Mo}$ and ${ }^{92} \mathrm{Mo}(\gamma, \mathrm{n}){ }^{91} \mathrm{Mo}$ reactions are presented. To verify the accuracy of the method, the results for the stable isotopes will be compared to the measurements with bremsstrahlung photons done at S-DALINAC (TU Darmstadt) and ELBE (FZ DresdenRossendorf).
\end{abstract}

11th Symposium on Nuclei in the Cosmos, NIC XI

July 19-23, 2010

Heidelberg, Germany

*This project is supported by the HGF Young Investigators Project VH-NG-327.

† Speaker. 


\section{Introduction}

Photo-dissociation reactions are important to explain the abundances of the nuclei produced via the so-called p-process. There are 32 stable neutron-deficient nuclei between ${ }^{74} \mathrm{Se}$ and ${ }^{196} \mathrm{Hg}$ which cannot be produced by neutron capture reactions in the framework of the $r$ - and s-processes. For the lighter p-nuclei, the dominating production mechanism is the proton capture. Heavier pnuclei are produced via a series of photo-disintegration reactions such as $(\gamma, \mathrm{n}),(\gamma, \mathrm{p})$ and $(\gamma, \alpha)$ on stable s- and r-process seed nuclei. For the p-process, temperatures of $2-3 \cdot 10^{9} \mathrm{~K}$ over a few seconds are required. Such conditions are commonly considered to take place in supernova explosions.

A long-standing mystery in the $A \sim 100$ mass region is that in all present models a significant underproduction of the p-nuclei ${ }^{92} \mathrm{Mo}$ and ${ }^{96} \mathrm{Ru}$ is observed. These isotopes are among the most abundant p-nuclei [1]. One possible source of this discrepancy might be an unreliability of the nuclear physics input used in the network calculations of isotopic abundances. Most of the reaction rates are predicted with the help of the Hauser-Feshbach statistical model [2], but the uncertainties of the model are quite large. It is therefore very important that as many reaction rates as possible are determined experimentally to provide a reliable reference for the calculations.

\section{Experimental method}

At the SIS/FRS/LAND facility at GSI, Coulomb dissociation (CD) experiments on the stable ${ }^{92,94,100}$ Mo and unstable ${ }^{93}$ Mo isotopes were performed in order to extract $(\gamma, \mathrm{n})$ and $(\gamma, 2 \mathrm{n})$ reaction cross sections. The $\mathrm{CD}$ technique implies inverse kinematics, which means that the nucleus under investigation serves as a projectile and impinges on a thick lead target. Under certain conditions, such as a sufficiently high energy and a large impact parameter, a reaction takes place between the projectile and a virtual photon originating from the Coulomb field of the target nucleus. One of the main advantages of this method is that it allows studies of very short-lived isotopes, which cannot be prepared as a target.

${ }^{94} \mathrm{Mo}$ and ${ }^{100}$ Mo primary beams were accelerated by the heavy ion synchrotron SIS and conducted directly to the experimental area (Cave C), where the LAND setup is located. Beams of neutron-deficient ${ }^{92} \mathrm{Mo}$ and radioactive ${ }^{93} \mathrm{Mo}$ were produced from a primary ${ }^{94} \mathrm{Mo}$ beam by the inflight fragmentation technique using the Fragment Separator (FRS) [3]. In all these cases, the energy of the beam at the cave entrance was approximately $500 \mathrm{MeV} / \mathrm{u}$.

Fig. 1 shows the sketch of the LAND experimental setup. It provides the possibility to detect all reaction products, thus delivering a kinematically complete measurement. The incoming particles are identified with respect to $A$ and $Z$ via time-of-flight and energy-loss measurements. Their magnetic rigidity is also required and is provided by the FRS. The time-of-flight is measured between two plastic scintillator detectors (S8 and POS) located at the exit of the FRS and at the beginning of the Cave $\mathrm{C}$ beam line, respectively. Energy-loss measurements are performed with two Si pin-diodes (PSP1,2), which provide position information as well. The reaction target is located at the entrance of a barrel-shaped $\gamma$-spectrometer consisting of 144 CsI crystals.

Behind the target, charged outgoing fragments are deflected by the field of a large gap dipole magnet (ALADIN), while the evaporated neutrons continue their flight in the forward direction and hit the LAND neutron detector [5], where their energies, arrival times and positions are measured. 


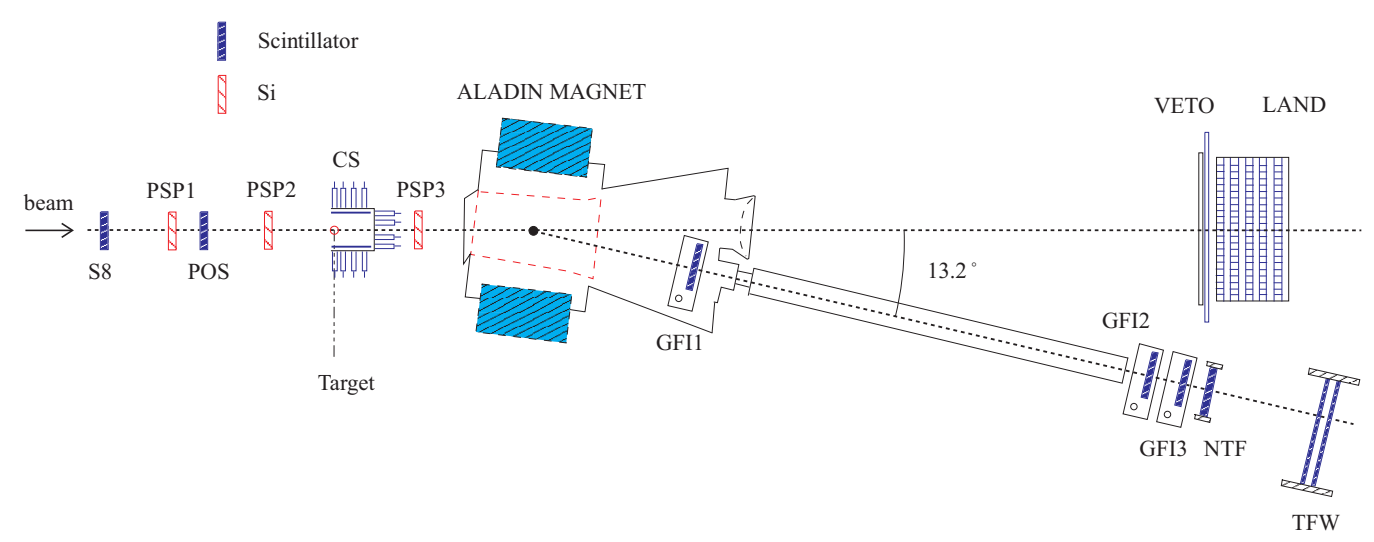

Figure 1: LAND experimental setup (from the $\mathrm{PhD}$ thesis of S. Paschalis [凹]).

Outgoing fragments are identified in a similar manner as the incoming ions. The fragment charge is determined from a combination of two energy-loss measurements: in the PSP3 detector located behind the target and the time-of-flight wall (TFW) at the end of the fragment track. A 2-dimensional cut on this correlation allows to reject events where the fragment broke up on its way to the TFW. In order to determine the mass, the fragment is tracked through the ALADIN field, which requires at least three position measurements (two before and one after the magnet or vice versa). These measurements are available from three fiber detectors (GFI1,2,3) located in the fragment arm after the magnet, PSP3 in front of the magnet and also PSP1 and PSP2, which allow to reconstruct the position of the ion on the target.

\section{Analysis and preliminary results}

In order to extract the electromagnetic excitation cross sections from the measurement with the $\mathrm{Pb}$ target, a contribution of the following background reactions has to be taken into account: nuclear reactions at the target and all kinds of reactions outside the target. The first contribution is estimated from an additional measurement with a carbon target, the second one from a measurement without any target. Both background contributions are appropriately scaled and subtracted from the $\mathrm{Pb}$ target data.

A clean selection of the reaction channel is ensured by a proper set of cuts. This includes a 2-dimensional cut on the incoming ion in the $A / Z$ vs $Z$ plane, a 2-dimensional cut on the outgoing $Z$, a cut on neutron multiplicity in LAND corresponding to the channel of interest and a cut on neutron velocity in LAND, which helps to reject the background from other detectors located near LAND. The mass distribution of the outgoing fragments was obtained with the above-mentioned procedure and is shown in Fig. 2 for three reaction channels: ${ }^{100} \mathrm{Mo}(\gamma, \mathrm{n}){ }^{99} \mathrm{Mo},{ }^{100} \mathrm{Mo}(\gamma, 2 \mathrm{n}){ }^{98} \mathrm{Mo}$ and ${ }^{92} \mathrm{Mo}(\gamma, \mathrm{n}){ }^{91} \mathrm{Mo}$. Apart from the main peak corresponding to the expected $A$, a contamination from the neighboring masses is always present, which is a result of wrongly reconstructed neutrons in LAND. A mass resolution of $\sigma=0.38$ mass units does not allow the neighboring masses to be well resolved, so a mass cut determined from this distribution has to be rather narrow $( \pm 1 \sigma)$ and the efficiency of this cut, as well as the amount of contamination, must be carefully estimated. 

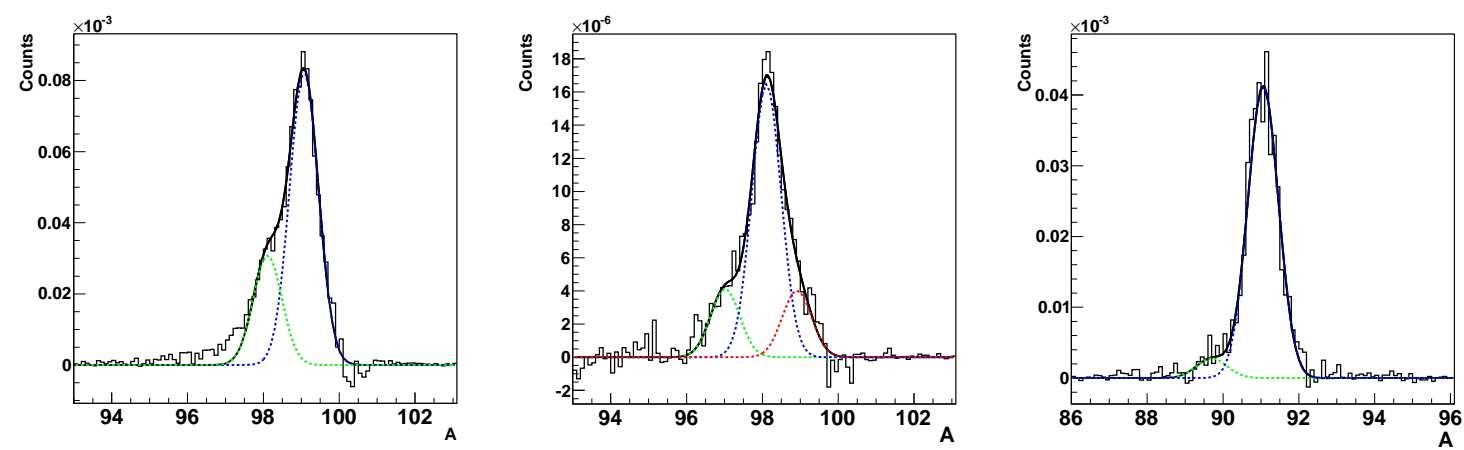

Figure 2: Mass distributions of the outgoing fragment after tracking through the magnet for three reaction channels. Left: ${ }^{100} \mathrm{Mo}(\gamma, \mathrm{n}){ }^{99} \mathrm{Mo}$; middle: ${ }^{100} \mathrm{Mo}(\gamma, 2 \mathrm{n}){ }^{98} \mathrm{Mo}$; right: ${ }^{92} \mathrm{Mo}(\gamma, \mathrm{n}){ }^{91} \mathrm{Mo}$.

\begin{tabular}{|c|c|c|c|}
\hline Reaction & ${ }^{100} \mathrm{Mo}(\gamma, \mathrm{n}){ }^{99} \mathrm{Mo}$ & ${ }^{100} \mathrm{Mo}(\gamma, 2 \mathrm{n}){ }^{98} \mathrm{Mo}$ & ${ }^{92} \mathrm{Mo}(\gamma, \mathrm{n}){ }^{91} \mathrm{Mo}$ \\
\hline$\sigma_{C E}($ this work, preliminary), mb & 812 & 285 & 437 \\
\hline$\sigma_{C E}($ Beil et al. [阿), mb & $876 \pm 30$ & $293 \pm 20$ & \\
\hline
\end{tabular}

Table 1: Integrated Coulomb excitation cross sections for the three reaction channels

Finally, the efficiency and acceptance of LAND have to be taken into account. The nominal LAND efficiency was determined from an earlier LAND calibration experiment and amounts to 94\% for $500 \mathrm{MeV}$ neutrons. Additional effects, such as switched-off paddles, effective thresholds and the acceptance of LAND were simulated. They strongly depend on the sum of the kinetic energies of the neutrons and, e.g., for one neutron the total efficiency drops from $79 \%$ at $1 \mathrm{MeV}$ to $40 \%$ at $5 \mathrm{MeV}$ kinetic energy (in the rest frame of the emitting nucleus) [6]. These effects must be corrected for on an event-by-event basis: each event which passed the cuts receives a statistical weight based on the total kinetic energy of the neutrons.

Preliminary integral Coulomb excitation cross sections for the three reaction channels are presented in Table 1. Statistical uncertainties of the data are less than $3 \%$, but a non-negligible systematic uncertainty is present and is estimated at approximately $10 \%$.

Although the setup provides a possibility to reconstruct the excitation energy spectrum via the invariant mass method, difficulties were encountered on the way to understand the efficiency of the CsI $\gamma$-detector, which appeared to be unexpectedly low. The overall response of the setup (influenced mainly by LAND and the CsI detector) is very complex and does not allow a deconvolution in order to reconstruct the differential cross section. One of the possible approaches to the problem using a dedicated event generator was extensively studied in the $\mathrm{PhD}$ thesis of $\mathrm{D}$. Rossi [6].

\section{Comparison to photoabsorption data}

Integrated Coulomb excitation cross sections for ${ }^{100} \mathrm{Mo}$ were compared to the measurement by Beil et al. 诃 after convoluting the latter with the E1 component of the virtual photon spectrum (see Table 1). E2 contribution to the integral cross section was estimated at approximately $10 \%$. Beil et al. used a monoenergetic photon beam produced by positron annihilation to measure the following total photoabsorption cross sections: $\sigma_{\gamma}(\gamma, n)=\sigma[(\gamma, n)+(\gamma, p n)]$ and $\sigma_{\gamma}(\gamma, 2 n)=$ 
$\sigma[(\gamma, 2 n)+(\gamma, p n n)]$. In our data, the proton removal channels are clearly separated and are not analyzed. However, in the case of ${ }^{100} \mathrm{Mo}$, the proton removal reaction threshold is high enough to neglect the contribution of this channel. In the case of ${ }^{92} \mathrm{Mo}(\gamma, n)$, a direct comparison was not possible due to the low $(\gamma, \mathrm{np})$ reaction threshold.

It should be noted that the photoabsorption data [7] might need an additional scaling by a factor 0.85, as suggested by Berman et al. [8]. Erhard et al. [9] showed that their activation data on ${ }^{100} \operatorname{Mo}(\gamma, \mathrm{n})$ agree with [7] if scaled by $0.89 \pm 0.04$. Berman et al. [8] assume that the discrepancy might come from a wrong estimation of the photon flux and/or neutron detection efficiency in [7]. We will try to contribute to the understanding of this controversy.

\section{Summary and outlook}

The Coulomb excitation cross sections on the stable ${ }^{92,94,100}$ Mo and unstable ${ }^{93}$ Mo isotopes were measured at the SIS/FRS/LAND facility at GSI. Preliminary integral cross sections for ${ }^{100} \mathrm{Mo}(\gamma, \mathrm{n})$, ${ }^{100} \operatorname{Mo}(\gamma, 2 \mathrm{n})$ and ${ }^{92} \mathrm{Mo}(\gamma, \mathrm{n})$ reactions are presented, which prove the feasibility of the method. An important aspect of the project is to verify the accuracy of the CD method by comparing the data with direct $(\gamma, \mathrm{n})$ experiments performed with real photons at S-DALINAC (TU Darmstadt) and ELBE (FZD) [9].

The data analysis is in process, eventually leading to Coulomb excitation cross sections for the ${ }^{94} \mathrm{Mo}$ and ${ }^{93} \mathrm{Mo}$ isotopes. The latter is of particular interest, since it is unstable and its cross section was never measured before.

\section{References}

[1] M. Arnould and S. Goriely. The p-process of stellar nucleosynthesis: astrophysics and nuclear physics status. Physics Reports 384 (2003) 1.

[2] T. Rauscher and F.-K. Thielemann. Astrophysical Reaction Rates From Statistical Model Calculations. Atomic Data and Nuclear Data Tables 75(1-2) (2000) 1.

[3] H. Geissel, P. Armbruster et al. The GSI projectile fragment separator (FRS): a versatile magnetic system for relativistic heavy ions. NIM B 70(1-4) (1992) 286.

[4] S. Paschalis. Relativistic One-Nucleon Removal Reactions. Ph.D. thesis, University of Liverpool, UK (2008).

[5] T. Blaich, T. W. Elze et al. A large area detector for high-energy neutrons. NIM A 314(1) (1992) 136.

[6] D. M. Rossi. Investigation of the Dipole Response of Nickel Isotopes in the Presence of a High-Frequency Electromagnetic Field. Ph.D. thesis, Johannes Gutenberg-Universität Mainz, Germany (2010).

[7] H. Beil, R. Bergère et al. A study of the photoneutron contribution to the giant dipole resonance in doubly even Mo isotopes. Nucl. Phys. A 227(3) (1974) 427.

[8] B. L. Berman, R. E. Pywell et al. Absolute photoneutron cross sections for $\mathrm{Zr}, \mathrm{I}, \mathrm{Pr}$, Au, and Pb. Phys. Rev. C 36(4) (1987) 1286.

[9] M. Erhard, A. R. Junghans et al. Experimental study of the electric dipole strength in the even Mo nuclei and its deformation dependence. Phys. Rev. C 81(3) (2010) 034319. 